\title{
$\underline{\mathbf{P}-98}$
}

\section{Chemical Composition and Antioxidant Properties of Backhousia Citriodora Volatile Oil}

\author{
Ong Boo Kean", Norulaiman Yusoff, Nor Azah Mohamad Ali, Vimala Subramaniam and Sam Kar \\ Yee \\ Natural Products Division, Forest Research Institute Malaysia FRIM, 52109 Kepong, Selangor, Malaysia; \\ E-mail: ongbk@frim.gov.my
}

Backhousia citriodora commonly known as lemon myrtle belongs to Myrtaceae family. This species is an evergreen tree which can grow up to 30 meters. Lemon myrtle is highly aromatic, having an exquisite flavour and aroma of a blend of lemongrass, lime and lemon. The leaf and flowers are used in tea blends and beverages, dairy, biscuits, breads, confectionery, pasta, syrups, liqueurs, flavoured oils, dipping and simmer sauces. In our study, the chemical composition and antioxidant properties of the lemon myrtle volatile oil obtained via hydro distillation were investigated. Examination by gas chromatography (GC) and gas chromatography/mass spectrometry (GC/MS) analysis showed that the volatile oil obtained from the leaves was made up predominantly neral $(39.57 \%)$ and geranial $(52.43 \%)$. Linalool $(0.37 \%)$, citronellal $(0.15 \%)$, E-isocitral $(2.47 \%)$, geraniol $(0.66 \%)$, ethyl geranate $(0.13 \%)$, caryophyllene $(0.08 \%)$ and spathulenol $(0.09 \%)$ were the other component present in significant amounts. For the stem part, similarly the major components also are neral (36.76\%) and geranial $(56.30 \%)$. Other minor components found in stem oil were 6-methyl-5-hepten-2-one $(0.13 \%)$, linolool $(0.24 \%)$, citronellal $(0.09 \%)$, trans-paramentha-2,8-dien-1-ol (0.39\%), E-isocitral (0.92\%), (3Z)-hexenyl-(3Z)-hexenoate (0.22\%), 8-hydroxy-neo-menthol (1.17\%), (E)- $\alpha$-Damascene $0.70 \%)$, spathulenol $(0.17 \%)$ and globulol $(0.14 \%)$. The obtained volatile oils were screen for the antioxidant properties by using DPPH (1,2-diphenyl-2-picryl-hydrazyl) radical scavenging assay and xanthine/xanthine oxidase superoxide scavenging assay. Our study indicated both the lemon myrtle leaf and stem volatile oil showed high antioxidant properties. DPPH scavenging activity for both the leaf and stem parts are $83.72 \%$ and $84.46 \%$ respectively. As for the xanthine/ xanthine oxidase superoxide scavenging assay, both the lemon myrtle leaf and stem volatile oil showed high superoxide scavenging activity, which are $83.72 \%$ and $84.46 \%$ respectively.

Keywords: Backhousia citrodora, lemon myrtle, chemical constituents, antioxidant. 